\title{
Impacto de la implementación de la prueba rápida del VIH/ITS en un centro de atención especializada en Brasil*
}

\section{Impact of the Implementation of the Rapid HIV/STI Test in a Specialized Care Center in Brazil}

\section{Impacto da implementação de teste rápido do VIH/ITS em um centro de atenção especializada no Brasil}

Fecha de recepción: 27-06 2016 Fecha de aceptación: 13-03-2017 Disponible en línea: 30-05-2017 doi:10.11144/Javeriana.rgps16-32.iipr

Cómo citar este artículo:

Rocha KB, Carvalho FT, Weber JLA, Both NS, Pizzinato A. Impacto de la implementación de la prueba rápida del VIH/ITS en un centro de atención especializada en Brasil. Rev Gerenc Polít Salud. 2016; 16 (32): 108-119. https://doi.org/10.11144/Javeriana.rgps16-32.iipr

\author{
Kátia Bones-Rocha** \\ Fernanda Torres de Carvalho*** \\ João Luís Almeida-Weber**** \\ Nalu Silvana-Both***** \\ Adolfo Pizzinato******
}

\footnotetext{
* $\quad$ Artículo de investigación. Proyecto de investigación Prueba rápida para el VIH, sífilis y hepatitis viral: análisis del impacto de esta tecnología en el acceso de poblaciones más vulnerables en un Centro de Atención Especializada de Porto Alegre, Brasil. Inicio: octubre de 2013; finalización: diciembre de 2015. Pontifícia Universidade Católica do Rio Grande do Sul. Proyecto financiado por el Ministerio de salud MSBRAK-57 (licitación para la selección de subproyectos de investigación en ITS, VIH/SIDA y hepatitis virales 01/2013). Instituiciones ejecutoras: Pontifícia Universade Católica do Rio Grande do Sul, en colaboración con el Ambulatório de Dermatologia Sanitária del Rio Grande do Sul. Correo electrónico: katiabonesrocha@gmail.com

* Profesora, doctora en Psicología, investigadora en la Pontifícia Universidade Católica do Rio Grande do Sul, Porto Alegre (Brasil). Correspondencia: Av. Ipiranga, 6681 - Prédio 11, sala 931 - Programa de Pós-Graduação em Psicologia - Pontifícia Universidade Católica do Rio Grande do Sul (PUCRS). Porto Alegre (RS) - Brasil. Correo electrónico: katia.rocha@pucrs.br

*** Psicóloga, doctora en Psicología, psicóloga en el Ambulatório de Dermatologia Sanitária de la Secretaria Estadual de Saúde do Rio Grande do Sul, Porto Alegre (Brasil). Correo electrónico: torresdecarvalho@yahoo.com.br

**** Psicólogo, magíster en Psicología, investigador en la Pontifícia Universidade Católica do Rio Grande do Sul, Porto Alegre (Brasil). Correo electrónico: joao.weber@acad.pucrs.br

***** Psicóloga, magíster en Sociología, psicóloga en el Ambulatório de Dermatologia Sanitária de la Secretaria Estadual de Saúde do Rio Grande do Sul, Porto Alegre (Brasil). Correo electrónico: naluboth@gmail.com

****** Profesor, doctor en Psicología Social, investigador en la Pontifícia Universidade Católica do Rio Grande do Sul, Porto Alegre (Brasil). Correo electrónico: adolfo.pizzinato@pucrs.br
} 


\section{Resumen}

Objetivo: evaluar el impacto de la implementación de la consejería y prueba rápida del VIH e infecciones de transmisión sexual en un centro especializado de consejería y diagnóstico de VIH (CE). Método: estudio analítico con un grupo de intervención y un grupo control en el cual fueron comparadas las medias mensuales de las pruebas y consejerías realizadas entre un centro especializado que implementó la prueba rápida de VIH para todos los pacientes (CE caso) y otro centro especializado que utiliza la modalidad tradicional de la prueba (diagnóstico en treinta días) (CE control). Resultados: el CE (caso) tuvo un aumento significativo en el número de pruebas realizadas entre los dos periodos. En el CE (caso) hubo una disminución en la proporción de personas de la población general que se hicieron la prueba y un aumento en las personas más vulnerables al VIH. Conclusiones: la implementación de la prueba rápida tuvo un impacto positivo en el número de pruebas realizadas y también en el acceso a las personas en situación de vulnerabilidad.

Palabras clave: sida; VIH; ITS; atención de salud; políticas públicas en salud; calidad, acceso y evaluación de la atención de salud

\section{Abstract}

Objective: to evaluate the impact of the implementation of counseling and rapid testing of HIV and sexually transmitted infections in a specialized HIV counseling and diagnosis center (CE). Method: analytical study with an intervention group and a control group; a comparison was established between the monthly means of tests and counseling sessions performed in a specialized center that implemented the HIV rapid test for all patients (case CE) and another specialized center which uses the traditional form of the test (diagnosis in thirty days) (control CE). Results: CE (case) had a significant increase in the number of tests performed between the two periods. In the CE (case) there was a decrease in the proportion of people in the general population who were tested and an increase in the people most vulnerable to HIV. Conclusions: the implementation of the rapid test had a positive impact on the number of tests carried out and also on the access provided to people in vulnerable situations.

Keywords: AIDS; HIV; health care; STIs; public health policies; health care quality, access and evaluation.

\section{Resumo}

Objetivo: Avaliar o impacto da implantação do aconselhamento e teste rápido de HIV e doenças sexualmente transmissíveis em um Centro de Testagem e Aconselhamento (CTA). Método: Estudo analítico com um grupo de intervenção e um grupo controle em que foram comparadas as médias mensais de Testagem e Aconselhamento realizada em um CTA (caso) que implementou o teste rápido de HIV para todos os pacientes e outro CTA (controle) que utiliza o teste tradicional de HIV em que o resultado fica disponível em 30 dias. Resultados: O CTA (caso) teve um aumento significativo no número de testes realizados entre os dois períodos. Em CTA (caso), houve uma diminuição na proporção de pessoas que realizaram o teste da população geral e o aumentou da realização dos testes pelas pessoas consideradas mais vulneráveis ao HIV. Conclusões: A implantação do teste rápido teve um impacto positivo sobre o número de testes realizados e acesso a pessoas em situação de vulnerabilidade.

Palavras chave: Aids; HIV; sistemas de saúde; Infecções sexualmente transmissíveis; políticas públicas de saúde; qualidade do sistema de saúde; acesso e avaliação 


\section{Introducción}

La prevalencia del VIH en Brasil es considerada entre baja y moderada, con 730000 habitantes portadores $(0.5 \mathrm{a} 1 \%)$. La incidencia anual fue de 20.1/100000 casos en 2011 (1); sin embargo, la incidencia es mayor en algunas regiones de Brasil. El estado de Rio Grande do Sul y su capital Porto Alegre continúan notificando elevados casos de sida en el escenario epidemiológico brasilero, con un índice de incidencia de 40,2/100000 habitantes. Porto Alegre registra 93,7/100000 habitantes, siendo este un índice de los más altos entre las capitales brasileras (2).

Desde el inicio de la epidemia del sida, Brasil ha implementado un abordaje innovador para enfrentarla, basado en el compromiso político y la evidencia científica y contribuyendo a la comprensión de la epidemia a partir de la perspectiva de los derechos humanos (1). Uno de los desafíos de la epidemia en Brasil es la alta incidencia de inicio tardío del tratamiento. La literatura nacional e internacional destaca que tardar en el inicio del tratamiento facilita el desarrollo de la enfermedad, aumenta el índice de mortalidad, eleva el número de transmisiones del virus y encarece el tratamiento para el Estado (3-4-5). En Brasil, aproximadamente, $41 \%$ de las personas inician el tratamiento en estado avanzado de la enfermedad (6), lo que está asociado al desconocimiento de la condición serológica, la dificultad de acceso al diagnóstico y a la atención en los servicios de salud, así como a la percepción de la población que no se ve en riesgo o siente miedo de ser discriminada debido al diagnóstico (6).

Personas que viven en situación de mayor vulnerabilidad, definidos por el Ministerio de la Salud de Brasil como poblaciones-cla110 ve (1), presentan mayor prevalencia de sida. Por ejemplo, en el periodo 2009-2010 se identificó una prevalencia de esta enfermedad de $10.5 \%$ en hombres que tenían sexo con hombres (HSH), $5.9 \%$ en usuarios de drogas y $4.9 \%$ en profesionales del sexo (1). Estudios recientes desarrollados en Porto Alegre muestran una prevalencia de $25 \%$ entre transexuales (7). Estudios anteriores muestran que estas poblaciones tienen dificultad para acceder a los servicios de salud $(8,9)$. Probablemente por esa razón, pacientes con opciones sexuales no hegemónicas, como travestis, transgénero, jóvenes gays y profesionales del sexo prefieren muchas veces ser atendidos por centros especializados, como los Centros de Consejería y Prueba del VIH (CE) $(8,9)$. Cabe resaltar que en Brasil hace parte de la misión de los CE priorizar la atención a los sectores poblacionales en situación de mayor vulnerabilidad, así como promover la equidad y el acceso a la consejería, al diagnóstico del VIH, hepatitis B y C y sífilis y a la prevención de estas y demás infecciones de transmisión sexual (ITS) (10).

Para incentivar la detección precoz de la infección y el inicio del tratamiento del VIH, a partir de 2001, en Brasil se difundieron recomendaciones del Ministerio de Salud para inclusión de la prueba rápida del VIH y aumentó la oferta de la prueba en diferentes niveles de atención. Esta política sigue las recomendaciones de la Organización Mundial de la Salud (OMS), de los United States Centers for Disease Control and Prevention y de la French Haute Autorité de Santé $(11,12)$. Diferentes estudios evalúan la costoefectividad de la prueba rápida del VIH en diferentes países $(13,14)$, algunos reforzando la efectividad de esa oferta para toda la población $(13,15)$ y otros sugiriendo focalizarse en los grupos considerados más vulnerables (14).

En un estudio realizado por Wouters et al. (16) con una muestra de 5025 participantes, 
la introducción de la prueba rápida fue altamente satisfactoria entre las poblaciones vulnerables. En otro estudio se recomienda la prueba rápida de rutina para todos los adultos, excepto en localidades donde hay evidencia de que la prevalencia del VIH esta debajo de $0.2 \%$ (13). En Brasil la implementación de la prueba rápida se justifica por su eficiencia y confiabilidad, pero también por su relación costo-beneficio. Otra ventaja es la posibilidad de ofrecer acogida inmediata a los portadores del VIH dentro del Sistema Único de Salud (SUS). En Brasil en el momento de la realización de la prueba del VIH y otras ITS el paciente es atendido por un profesional de salud, antes y después de la prueba, quien realiza la consejería en relación con el VIH y otras ITS.

En el estado de Rio Grande do Sul, el CE del Ambulatorio de Dermatología Sanitaria de Porto Alegre (CE/ADS) fue pionero en la oferta universal de la prueba rápida para el VIH, sífilis y hepatitis B y C para todos los pacientes, a partir de octubre de 2012. De esta manera, se hace necesaria la evaluación del impacto de esa estrategia para el servicio y para la comunidad atendida. En el presente estudio se buscó examinar ese impacto a partir de tres objetivos: 1) comparar el número de personas que han realizado la consejería y prueba rápida del VIH y otras ITS en el $\mathrm{CE} / \mathrm{ADS}$ (caso), en el periodo de doce meses anteriores y posteriores a la implementación de la políticas de la prueba rápida; 2) comparar el número de personas que han realizado la consejería y prueba rápida del VIH y otras ITS por el CE/ADS (caso) con la atención realizada por el otro CE (control) de la ciudad, cuya estrategia prioritaria sigue siendo la modalidad tradicional de la prueba (diagnóstico en treinta días); y 3 ) investigar si la prueba rápida (diagnóstico en treinta minutos) puede o no haber estimulado a las poblaciones en situación de mayor vulnerabilidad a realizarse la prueba.

\section{Método}

Se trata de un estudio analítico con un grupo de intervención y un grupo control (17). En la muestra se incluyó a todas las personas atendidas en el Centro Especializado de Consejería y Prueba del VIH del Ambulatorio de Dermatología Sanitaria de Rio Grande do Sul CE/ADS (caso) en Porto Alegre, en el periodo de octubre de 2011 a septiembre de 2013. Se trata de un periodo de doce meses anteriores y de los doce meses posteriores a la implementación de la política de oferta universal de pruebas rápidas en el servicio que inició en octubre del 2012.

El CE/ADS (caso), el primer CE de Brasil, fue creado por el Ministerio de Salud en 1988. Tiene su sede en el ADS que funciona en Porto Alegre desde 1920, históricamente destinado al tratamiento de problemas dermatológicos, lepra y enfermedades de trasmisión sexual, a lo que se sumó en la década de los ochenta la atención a los primeros casos de sida del país (10). En este sentido, el CE/ADS funciona como una importante referencia en la capital y en el estado para el diagnóstico y el tratamiento de ITS/VIH/ sida. El número promedio anual de pacientes que realizaron la consejería y la prueba de VIH/ITS en el CE/ADS en el periodo de 2011 a 2016 fue de 2946.

Los datos para el presente estudio fueron obtenidos del sistema nacional de información de CE-SI-CE, que reúne las informaciones de la prueba serológica, además de las respuestas de un formulario estándar aplicado a todos los pacientes del servicio. Para el estudio de tendencia se examinaron los números mensuales de atenciones realizadas en el CE/ADS (caso) en los periodos pre y posimplementación de la política de pruebas rápidas.

Entre las variables utilizadas está el número de pruebas realizadas en el periodo. 
Asimismo, en el CE/ADS (caso) se analizó a que grupos poblacionales pertenecían las personas que realizaron la prueba convencional (preimplementación) y la prueba rápida (posimplementación). Con base en SI-CE, los participantes fueron agrupados en: portadores de ITS; compañeros(as) de VIH positivo; hombres que tiene sexo con hombres (HSH); profesionales del sexo; personas que utilizaron alguna droga en los últimos doce meses; usuarios de drogas ilícitas frecuentes, usuarios frecuentes de alcohol.

Para tener una muestra pequeña de algunos de estos grupos más vulnerables, fueron agrupados en una variable denominada población clave, la cual reúne: población confinada, profesionales del sexo, HSH, usuarios de drogas, portador de ITS, travesti/transexual, persona en exclusión social y portador de hepatitis B/C. Además, se incluyó una variable de poblaciones clave por dos o más criterios. Las variables de agrupamiento no son excluyentes entre sí; un mismo individuo puede pertenecer a más de un grupo.

Para el estudio de comparación entre los dos grupos, se estableció una comparación entre las medias mensuales de atención en el CE/ ADS (caso) y las medias mensuales del CE (control). De esta manera, se intentó verificar si las eventuales diferencias encontradas en los periodos pre y posimplementación de la política en el CE/ADS (caso) pudieron ser atribuidas al inicio de la oferta universal de las pruebas rápidas o respondieron a otras acciones más amplias, ligadas a la política estadual o nacional de enfrentamiento de la epidemia.

El CE (control) fue escogido por mostrar semejanzas clave con el CE (caso): con sede en Porto Alegre, también dentro de un servicio históricamente referente al tratamiento de VIH/sida, en la periferia de la ciudad. Asimismo, los flujos de trabajo en cuanto a la oferta de la prueba eran similares, en lo que se refiere a la oferta de asesoría pre y posprueba, oferta de exámenes adicionales de sífilis y hepatitis B y C, con excepción de la política de oferta universal de pruebas rápidas. Las pruebas ofrecidas eran realizadas prioritariamente en la modalidad convencional, donde el paciente no recibía el resultado el mismo día. La misma fuente de información (SI-CE) fue utilizada para la obtención de los datos del CE (control), considerando los mismos periodos de atención.

La primera etapa de análisis de los datos fue la comparación de las medias mensuales de la prueba y consejería realizadas en los dos periodos dentro de cada servicio y entre servicios, mediante el cálculo de prueba t-student. Se verificó la normalidad y la homocedasticidad de los dados. Posteriormente, se hizo un análisis de tendencia describiendo el número de pruebas realizadas mes a mes en cada periodo. Por último, se llevaron a cabo pruebas chi-cuadrado, cálculos de Odds Ratio (OR) e intervalos de confianza (IC 95\%) para comparar el perfil de las personas atendidas en el CE/ADS (caso) antes y después a la implementación de las pruebas rápidas. Se comparó el número de atenciones de cada categoría poblacional previamente definida - población general, portadores de ITS, HSH, compañero(a) de VIH positivo, profesionales del sexo, usuarios frecuentes de drogas y alcohol, poblaciones clave y poblaciones clave por dos o más criterios- en cada periodo.

El presente estudio fue aprobado por los comités de ética en investigación de la Pontifícia Universidade Católica do Rio Grande do Sul y de la Escuela de Salud Pública del Estado de Rio Grande do Sul. Se aplicó el proceso de consentimiento informado a los participantes y se respetó la confidencialidad de los participantes. 


\section{Resultados}

En el estudio se incluyó a 4017 pacientes, los cuales realizaron la prueba rápida para $\mathrm{VIH}$ en el CE/ADS durante el periodo estudiado. Se atendió a 1195 en los doce meses posteriores y, adicionalmente, se incluyó a 2811 pacientes que hicieron la prueba en el CEcontrol. En los doce meses correspondientes al periodo anterior a la implementación de la política de pruebas rápidas, en el CE-caso fueron atendidas 1543 personas, en tanto que en los doce meses posteriores se atendió a 1268 personas.

Los dos CE son servicios especializados de la misma ciudad, de acceso público universal, con equipos de profesionales de salud de diferentes áreas que tienen la capacidad de atender a un número semejante de pacientes y que ofrecen la consejería y prueba rápida del VIH y otras ITS. Las características de las muestras del CE/ADS (caso) y CE (control) en los periodo pre y posimplementación van en la misma dirección, aunque no hayan podido ser contrastadas estadísticamente (los datos del CE control solo estuvieron disponibles como agregados). La mayoría de los pacientes son hombres ( $71 \%$ caso; $57 \%$ control), la mayoría de los pacientes se identifican como blancos (65\% caso; $78 \%$ control). En relación con el estado civil, la mayoría de pacientes son solteros $(58.3 \%$ caso; $47 \%$ control) o casados ( $28.2 \%$ caso; $47 \%$ control). La escolaridad se distribuye principalmente en las categorías de cuatro a siete años ( $26.5 \%$ caso; $17.4 \%$ control) y en relación con el uso de preservativo $35 \%$ (caso) y $34 \%$ (control) refieren no utilizarlo.

En relación con el periodo posimplementación de la prueba rápida en el CE/ADS (caso) se observa un patrón similar. La mayoría son hombres (72\% caso; $54 \%$ control) y los pacientes se identifican como blancos (63.5\% caso; $78.4 \%$ control). En relación con el estado civil, la mayoría de los pacientes son solteros $(53.7 \%$ caso; $48.7 \%$ control) o casados ( $32 \%$ caso; $38.9 \%$ control), tienen entre ocho y once años de escolaridad (51.4\% caso; $41.1 \%$ control) y doce o más de escolaridad (23.1\% caso; $34.9 \%$ control). Con respecto al uso de preservativo, $37 \%$ (caso) y $33 \%$ (control) refieren no utilizarlo.

En cuanto a la comparación del número de pruebas pre y posimplementación de la prueba rápida (véase tabla 1 ), se identifica un aumento significativo en las medias mensuales de atención del CE/ADS (caso) cuando se comparan los periodos. En el periodo preimplementación, el CE (control) tenía una media mensual significativamente superior de atenciones $(m=131.75)$, en comparación con el CE/ADS (caso) $(\mathrm{m}=82.25)$. En relación con el periodo posimplementación, se identificó que se invirtieron los datos; en el CE/ADS (caso) se registró una media mensual de atenciones significativamente mayor $(\mathrm{m}=228.58)$. 
Tabla 1. Medias mensuales de personas atendidas para pruebas de VIH, CON COMparaciones entre los CE CASO Y CONTROL Y COMPARACIONES EN CADA SERVICIO ENTRE LOS PERIODOS PRE Y POSIMPLEMENTACIÓN DE LA POLÍTICA DE PRUEBAS RÁPIDAS EN EL CE/ADS (CASO)

\begin{tabular}{|l|c|c|c|c|c|c|c|}
\hline \multicolumn{1}{|c|}{ Local } & \multicolumn{2}{|c|}{2012} & \multicolumn{2}{|c|}{2013} & \\
\hline & $\begin{array}{c}\text { Pruebas } \\
\text { totales }\end{array}$ & $\begin{array}{c}\text { Media de } \\
\text { pruebas }\end{array}$ & $\begin{array}{c}\text { Desviación } \\
\text { estándar }\end{array}$ & $\begin{array}{c}\text { Media de } \\
\text { pruebas }\end{array}$ & $\begin{array}{c}\text { Desviación } \\
\text { estándar }\end{array}$ & p valor \\
\hline CE control & 2811 & 131.75 & 24.65 & 113.83 & 19.81 & $\mathrm{p}<.001$ \\
\hline $\begin{array}{l}\text { CE/ADS } \\
\text { caso }\end{array}$ & 4017 & 82.25 & 30.22 & 228.58 & 28.03 & $\mathrm{p}<.001$ \\
\hline & & & & & \\
\hline p valor & & $\mathrm{p}<.001$ & & $\mathrm{p}<.001$ & & \\
\hline
\end{tabular}

Fuente: elaboración propia con base en los datos del Ambulatorio de Dermatología Sanitaria(ADS) de Porto Alegre

La tabla 2 presenta la comparación del perfil de las personas atendidas en el CE/ADS antes y después de la implementación de las pruebas rápidas. En la tabla 2 se compara la distribución del número de las pruebas realizadas pre y posimplementación de la prueba rápida en el CE (caso) en algunas poblaciones clave. Los datos de la tabla 2 muestran que en los periodos pre y posprueba hubo una disminución significativa en el número de pruebas en la población general $(\mathrm{OR}=0.51$, $\mathrm{IC}=0.43-0.60)$, compañeros de personas portadoras de VIH y HSH. Por otro lado, las personas consideradas de poblaciones claves tuvieron una oportunidad mayor de hacerse la prueba $(\mathrm{OR}=1.19, \mathrm{IC}=1.19-1.39)$. Este aumento también fue significativo entre las personas portadoras de alguna ITS, usuarios de drogas en los últimos doce meses y usuarios de drogas ilícitas y alcohol frecuentes.

Tabla 2. Perfil de los pacientes atendidos en el CE/ADS, comparando los periodos PRE Y POSIMPLEMENTACIÓN DE LAS PRUEBAS RÁPIDAS

\begin{tabular}{|l|c|c|c|c|c|c|c|}
\hline & \multicolumn{2}{|c|}{ Pre } & \multicolumn{2}{c|}{ Pos } & \multicolumn{2}{l|}{} \\
\hline & $\mathrm{N}$ & $\%$ & $\mathrm{~N}$ & $\%$ & OR & $95 \%$ IC & $\mathrm{p}$ \\
\hline Población general & & & & & $0.51^{*}$ & & \\
\hline Sí & 873 & 77.4 & 1840 & 63.7 & & & \\
\hline No & 255 & 22.6 & 1049 & 36.3 & & & \\
\hline & & & & & & $0.43-0.6$ & $<.001$ \\
\hline Poblaciones clave $^{\text {a }}$ & & & & & $1.19^{*}$ & & \\
\hline Sí & 309 & 27.4 & 895 & 31.0 & & & \\
\hline No & 819 & 72.6 & 1994 & 69.0 & & & \\
\hline & & & & & & $1.19-1.39$ & $<.001$ \\
\hline Portador de ITS & & & & & $1.17^{*}$ & & \\
\hline Sí & 348 & 30.9 & 990 & 34.3 & & & \\
\hline No & 780 & 69.1 & 1899 & 65.7 & & & \\
\hline & & & & & & $1.01-1.35$ & $<.001$ \\
\hline
\end{tabular}




\begin{tabular}{|l|c|c|c|c|c|c|c|}
\hline Compañeros/as de VIH+ & & & & & $0.58^{*}$ & & \\
\hline Sí & 149 & 13.2 & 234 & 8.1 & & & \\
\hline No & 979 & 86.8 & 2655 & 91.9 & & & \\
\hline & & & & & & $0.47-0.72$ & $<.001$ \\
\hline HSH & & & & & $0.77^{*}$ & & \\
\hline Sí & 126 & 11.2 & 254 & 8.8 & & & \\
\hline No & 1002 & 88.8 & 2635 & 91.2 & & & \\
\hline & & & & & & $0.61-0.96$ & $<.001$ \\
\hline Profesionales del sexo & & & & & 1.48 & & \\
\hline Sí & 9 & 0.8 & 34 & 1.2 & & & \\
\hline No & 1119 & 99.2 & 2855 & 98.8 & & & \\
\hline & & & & & & $0.70-3.09$ & .380 \\
\hline Usuario drogas doce meses & & & & & $1.18^{*}$ & & \\
\hline Sí & 637 & 56.6 & 1745 & 60.4 & & & \\
\hline No & 491 & 43.5 & 1144 & 39.6 & & & \\
\hline & & & & & & $1.03-1.35$ & $<.001$ \\
\hline Usuario frecuente de alcohol & & & & & $1.36^{*}$ & & \\
\hline Sí & 62 & 5.5 & 212 & 7.3 & & & \\
\hline No & 1066 & 94.5 & 2677 & 92.7 & & & \\
\hline & & & & & & $1.02-1.82$ & $<.001$ \\
\hline Usuario frecuente de drogas & & & & & $1.68^{*}$ & & \\
\hline Sí & 37 & 3.3 & 156 & 5.4 & & & \\
\hline No & 1070 & 96.7 & 2733 & 94.6 & & & \\
\hline Sís criterios \\
No
\end{tabular}

a Poblaciones clave (población confinada, profesionales del sexo, HSH, usuarios de drogas, portador de ETS, travesti/transexual, persona en exclusión social, portador hepatitis $\mathrm{B} / \mathrm{C}$ ).

*Estadísticamente significativa

Fuente: elaboración propia con base en los datos del Ambulatorio de Dermatología Sanitaria (ADS) de Porto Alegre

La figura 1 muestra el número de pruebas realizadas por mes para cada una de los CTA. Se puede observar una tendencia muy similar de aumentos y disminuciones en el número de pruebas realizadas según los meses. Al comparar el periodo antes de la implementación de la prueba rápida de VIH — julio de 2011-junio de 2012- en el
CTA (caso) y en el mismo periodo en el control CE, en ambos se observa un aumento significativo en el mes de diciembre. Lo mismo ocurre en el periodo posterior a la ejecución —octubre de 2012-septiembre de 2013 - , en el cual hay un aumento en diciembre seguido de una disminución en los meses de enero y febrero. 


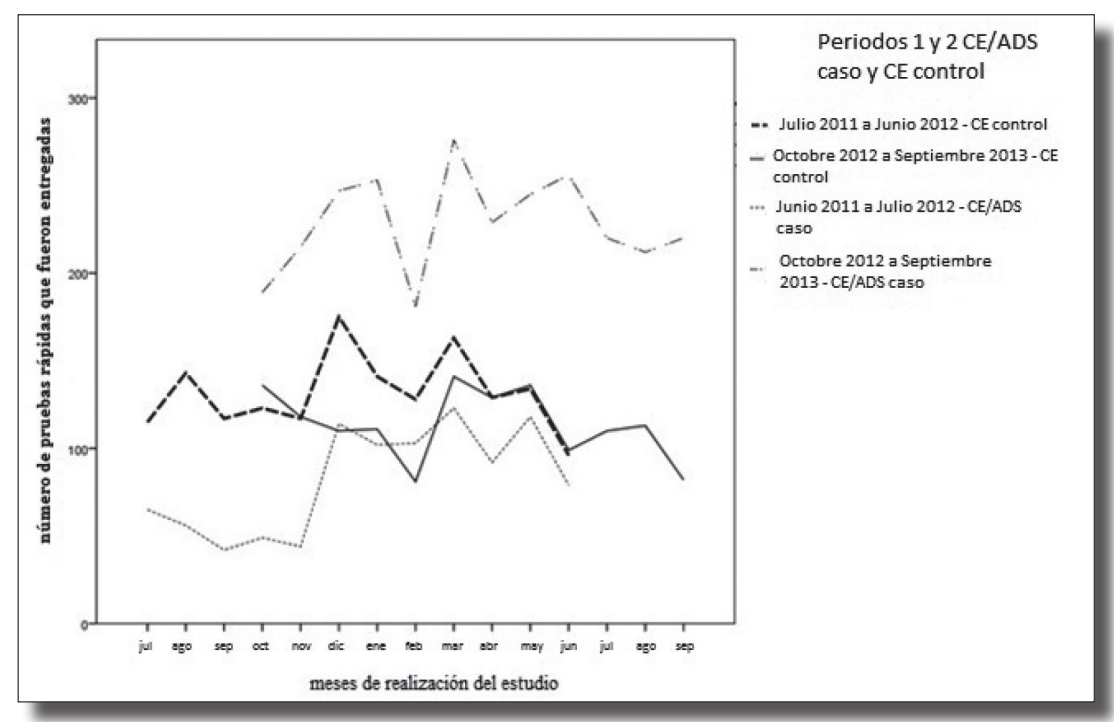

Figura 1. Descripción de la tendencia del número de Pruebas Realizadas mes a mes

Fuente: elaboración propia con base en los datos del Ambulatorio de Dermatología Sanitaria(ADS) de Porto Alegre

\section{Discusión}

La presente investigación buscó evaluar el impacto de la implementación de la prueba rápida para el VIH en el número de pruebas realizadas en un CE. Además de los resultados concretos del presente estudio, este representa un esfuerzo en el sentido de crear instrumentos y metodologías que permitan evaluar el impacto de las políticas implementadas en los servicios de salud.

Los resultados del presente estudio muestran que el CE (caso) que implementó la prueba rápida para todos los pacientes (CE/ADS) tuvo un aumento significativo en el número de pruebas realizadas entre el periodo de doce meses anteriores a la implementación y en los doce meses después de esta. Asimismo, se observa que este aumento fue estadísticamente superior al otro CE (control) que no adoptó la prueba rápida para todos los pacientes. Otro dato relevante es que en el CE/ADS (caso) hubo una disminución en el número de personas que se hicieron la prueba y que pertenecían a la población en general y, asimismo, un aumento en las poblaciones consideradas clave.

Esta disminución de la realización de pruebas para el VIH en la población en general puede estar relacionada con el aumento de la oferta de la prueba rápida dentro de la red de atención primaria, política que viene siendo implementada desde el año 2004 (18). El aumento de las pruebas en el CE entre las poblaciones en situación de mayor vulnerabilidad refuerza la importancia de los CE en la atención a poblaciones más vulnerables al VIH (10). En este sentido, se sugiere la expansión de la consejería y de la prueba rápida para otros $\mathrm{CE}$ del país, así como su adopción en otros países. Cabe resaltar que esta expansión debe estar aliada a la adopción de estrategias que vayan al encuentro de las necesidades de la población, desde una perspectiva contextualizada social y culturalmente, para disminuir las dificultades encontradas en la realización de la prueba (4). 
El aumento en la realización de la prueba rápida en poblaciones vulnerables en CE después de la implementación de la prueba rápida y de la consejería refuerza la importancia de esta estrategia, hecho que se describe en estudios internacionales $(13,16)$. Es importante destacar que existen diferentes niveles de vulnerabilidad a las ITS. El presente trabajo utiliza el concepto de vulnerabilidad y no el concepto de riesgo. Este último se asocia a la cuantificación de las posibilidades de padecimiento de individuos o poblaciones, a partir de las asociaciones entre eventos y condiciones patológicas, y eventos y condiciones no patológicas, causalmente relacionadas. Entre las limitaciones del concepto de riesgo está la falta de comprensión de las variables individuales dentro de la dinámica social, por lo cual se reducen los fenómenos a algunos de sus componentes que pueden ser medibles de forma separada. La idea de riesgo está asociada, exclusivamente, a las características de los individuos $(19,20)$.

Por otro lado, el concepto de vulnerabilidad tiene relación tanto con las condiciones individuales como con las condiciones institucionales y sociales (21). Estos tres componentes articulados priorizan el análisis y las intervenciones multidimensionales que consideran que las personas no son en sí vulnerables, pero pueden ser vulnerables a unos problemas y no a otros, en determinadas condiciones y diferentes momentos de sus vidas. La vulnerabilidad comprende los aspectos individuales, contextuales y programáticos que pueden contribuir a que las personas se vuelvan en algún momento de su vida más vulnerables (22). El presente estudio, a partir de datos cuantitativos, hace una aproximación a estos grupos que pueden ser considerados más vulnerables en función de la propia dificultad para acceder a los servicios de salud.

La posibilidad de ofrecer una acogida cualificada dentro del Sistema Único de Salud
(SUS) que consiga considerar las especificidades de cada persona en el proceso de atención, como ocurre en el caso de los CE, es una forma de dar una respuesta programática por parte del Estado a las diferentes necesidades de salud de la población. Como se afirmó anteriormente, algunas personas tienen dificultad para acceder a los servicios de salud debido a los prejuicios y a la discriminación que afirman sentir en los servicios de salud $(8,9)$. Así, se destaca la importancia de que los pacientes puedan acceder a los servicios de salud a partir de diferentes entradas, en las cuales puedan decidir como ciudadanos de derecho a cuál servicio acceder.

En este sentido, como destacan Perucchi et al. (23), la psicología puede hacer una importante contribución al análisis de las políticas de VIH/sida, una vez que se propone analizar los discursos que construyen las políticas de salud y que influyen en sus acciones, preguntándose qué sujetos estamos produciendo en estos espacios. Las autoras apuntan a la necesidad de cuestionar las verdades legitimadas por los discursos de poder que atraviesan las estrategias gubernamentales. El papel de la psicología se destaca en la elaboración y ejecución de las políticas públicas de VIH/sida y en la actuación más allá de una práctica asistencial, ayudando a la promoción de los derechos humanos y el ejercicio de ciudadanía $(4,23)$.

Como afirma Díaz-Bermúdez (24), formular políticas públicas basadas en evidencias científicas implica hacer cambios, no solamente asociados a la incorporación de mecanismos de participación social y de transferencia, sino también a atenuar la distancia que de tiempo atrás separa a la teoría de la práctica y a los sectores académicos de los prestadores de servicio como posibles actores de la formulación de estas políticas. De acuerdo con la autora, a partir de la experiencia cotidiana de los profesionales de servicios y sus 
interlocutores, se aprovecha una actuación renovada en los aspectos políticos, técnicos, gerenciales y humanos que conlleva el proceso de salud-enfermedad.

Los CE estudiados tienen una composición profesional y una oferta de servicios muy semejantes. Sin embargo, el presente estudio tiene como limitación no haber analizado si había diferencias estadísticamente significativas en el perfil de los pacientes entre los servicios, ya que por cuestiones de reserva y anonimato los datos de CE control estuvieron disponibles solamente de manera agregada, y así no teníamos los datos de cada paciente.

A partir de las reflexiones surgidas de este estudio, se propone la utilización de la presente metodología de estudio analítico con un grupo de intervención y un grupo control para la evaluación de la implementación de las políticas públicas en el territorio brasilero. Tal modelo permite comparar los resultados y genera indicadores que demuestran la eficacia o no de determinada acción, así como aquellos aspectos que pueden ser mejorados. Futuros estudios pueden investigar la implementación de la prueba rápida en otros $\mathrm{CE}$ de Brasil. Como punto fuerte del presente estudio es importante destacar que el área de evaluación de políticas públicas en Brasil y en otros países debe ser promocionada cada vez más.

\section{Agradecimientos}

Al Ministerio de Salud, que financió este proyecto por medio de la licitación para la selección de subproyectos de investigación en ITS, VIH/sida y hepatitis virales 01/2013; a la Coordinación Estadual de ITS/sida de Rio Grande do Sul; a todos los profesionales del Ambulatorio de Dermatología Sanitaria (ADS) de Porto Alegre, y también al CAPES, por la beca de posdoctorado concedida a una de las autoras (Proc. 10789/13-8).

\section{Referencias bibliográficas}

1. United Nations Programme on HIV/AIDS. A ONU e a resposta a Aids no Brasil [internet]. Brasília: Organização das Nações Unidas no Brasil; 2008 [acceso: 11 de octubre del 2014]. Disponible en: http://www.onubrasil.org.br/doc/LivretoUNAIDSCompleto.pdf

2. Ministério da Saúde. Boletim Epidemiológico - Aids e DST [internet]. Brasília: Secretaria de Vigilância em Saúde - Departamento de AIDS, DST e Hepatites virais; 2013 [acceso: 6 de octubre del 2014]. Disponible en: http://www.aids.gov.br/publicacao/2013/ boletim-epidemiologico-aids-e-dst-2013

3. Lazarini FM, Melchior R, González AD, Matsuo T. Tendencia de la epidemia de casos de sida en el Sur de Brasil en el período de 1986 a 2008. Rev Saúde Pública. 2012; 46(6):960-8.

4. Silva NEK, Oliveira LA, Sancho LG. Testagem anti-HIV: indagações sobre a expansão da oferta sob a perspectiva do acesso e da construção da demanda. Saúde em Debate. 2013;37(99):636-45.

5. Bassi SS, Martin Bland J, Martin F. High rates of fatality due to AIDS without universal HIV testing. J AIDS Clin Res. 2014;(5). doi:10.4172/21556113.100029

6. Ministério da Saúde. Material instrucional para Capacitação para profissionais multiplicadores para teste rápido HIV na plataforma DPP (Dual Parth Plataform) HIV e Sífilis e para Teste rápido para Hepatite B e C [internet]. Brasília: Secretaria de Vigilância em Saúde - Departamento de AIDS, DST e Hepatites virais; 2011 [acceso: 6 de octubre del 2014]. Disponible en: http://www.aids.gov.br/ pagina/2012/50770

7. Costa AB, Fontanari AMV, Jacinto MM, Silva DC, Lorencetti EK, Rosa Filho HT, et al. Population-based HIV prevalence and associated factors in male-to-female transsexuals from Southern Brazil. Arch Sex Behav. 2015; 44(2):521-4. doi:10.1007/ s10508-014-0386-Z

8. Cerqueira-Santos E, Calvetti PU, Rocha KB. Percepção de usuários gays, lésbicas, bissexuais, transgêneros, transexuais e travestis do Sistema Único de Saúde. Interam J Psychol. 2010; 44(2):235-45.

9. Rocha KB, Barbosa LH, Barboza CZ, Carvalho FT, Cerqueira-Santos E, Hermel JS, et al. Attitudes and perceptions of the Brazilian Public Health System by transgender individuals. Forum Qual Soc Res. 2009; 10(2):1-10.

10. Ministério da Saúde. Contribuição dos centros de testagem e aconselhamento para universalizar o diagnóstico e garantir a equidade no acesso aos serviços. Secretaria de Vigilância em Saúde. Programa Nacional de DST e Aids. Brasília: Secretaria de Vigilância em Saúde - Departamento de AIDS, DST e Hepatites virais; 2008. 
11. Haute Autorité de Santé. Dépistage de l'infection par le VIH en France: stratégies et dispositif de dépistage [internet]. Seine-Saint-Denis: Collège de la Haute Autorité de Santé; 2009 [acceso : 23 de septiembre del 2014]. Disponible en: http:// www.has-sante.fr/portail/upload/docs/application/ pdf/2009-10/synthese_depistage_vih_volet_2_ vfv_2009-10-21_16-48-3_460.pdf

12. World Health Organization. Scaling up HIV testing and counselling in the WHO European Region as an essential component of efforts to achieve universal access to HIV prevention, treatment, care and support: policy framework [internet]. Copenhagen: WHO European Region; 2008. [acceso en 2014 sept. 23]. Disponible en: http://www.who.int/hiv/ pub/vct/hiv_testing_counseling/en/

13. Paltiel AD, Walensky RP, Schackman BR, Seage GR, Mercincavage LM, Weintein MC, et al. Expanded HIV screening in the United States: effect on clinical outcomes, HIV, transmission, and costs. Ann Intern Med. 2006;145(11):797-806.

14. Yazdanpanah Y, Perelman J, Dilorenzo MA, Alves $\mathrm{J}$, Barros H, Mateus C. Routine HIV Screening in Portugal: Clinical impact and cost-effectiveness. PLoS ONE. 2013;8(12):e84173. doi:10.1371/journal.pone. 0084173

15. Plate DK. Evaluation and implementation of rapid HIV tests: the experience in 11 African countries. Aids Res Hum Retrov. 2011;23(12):1491-8.

16. Wouters K, Fransen K, Beelaert G, Platteau T, Ghyseghem CV, Collier I, et al. Use of rapid HIV test in low threshold center in Antwerp, Belgium, 2007-2012. Int J STD AIDS. 2014;25(13):936-42.
17. Robson C. Real world research: A resource for social sciences and practioner-researcher. Oxford: Blackwell; 1993.

18. Ministério da Saúde. Oficina de Aconselhamento em DST/HIV/AIDS para a atenção básica [internet]. Brasília: Secretaria de Vigilância em Saúde - Departamento de AIDS, DST e Hepatites virais; 2005 [acceso: 27 de septiembre del 2014]. Disponible en: http://bvsms.saude.gov.br/bvs/publicacoes/ acons_ind_atenbasica01_web.pdf

19. Almeida Filho N, Castiel LD, Ayres JR. Riesgo: concepto básico de la epidemiologia. Salud Colect. 2009; 5(3):322-44.

20. Zambenedetti G. Sala de espera como estratégia de educação em saúde no campo da atenção às doenças sexualmente transmissíveis. Saude Soc. 2012;21(4):1075-86.

21. Muñoz Sánchez AI, Bertolozzi MR. Pode o conceito de vulnerabilidade apoiar a construção do conhecimento em Saúde Coletiva?. Ciênc saúde coletiva. 2007;12(2):319-24.

22. Paiva VSF. Psicologia na saúde: sociopsicológica ou psicossocial? Inovações do campo no contexto da resposta brasileira à AIDS. Temas Psicol. 2013;21(3):531-49.

23. Perucchi J, Rodrigues FD, Jardim LN, Calais LB. Psicologia e políticas públicas em HIV/AIDS: algumas reflexões. Psicol Soc. 2011;23(1):72-80.

24. Díaz-Bermúdez XP. Uma questão de tempo: A implementação da estratégia do teste rápido para diagnóstico da infeccção de HIV no Brasil. En: Dhalia CBC, Díaz-Bermúdez XP, organizadores. Teste rápido - Por que não?. Brasília: Ministério da Saúde; 2007. p. 7-26. 